\title{
Metformin limits apoptosis in primary rat cortical astrocytes subjected to oxygen and glucose deprivation
}

\author{
Bożena Gabryel, Sebastian Liber \\ Department of Pharmacology, School of Medicine, Medical University of Silesia, Katowice, Poland
}

\begin{abstract}
Metformin, a type 2 anti-diabetic drug and an activator of AMP-activated protein kinase (AMPK), has been shown to reduce infarct size and pathological changes affecting astroglia in animal models of ischemic stroke. In this study, we evaluated how metformin affects cell viability, apoptosis and determined the role of AMPK, as well as JNK p46/ $p 54$ and $p 38$ kinases, in the observed phenomena in the culture of primary rat cortical astrocytes subjected to $12 \mathrm{~h}$ of oxygen and glucose deprivation (OGD). Metformin improved cell viability, reduced the fraction of apoptotic nuclei, and inhibited the activation of the executive caspase-3. Decreased activation of JNK p54 and p38 was associated with increased $B C-X_{L}$ expression and decreased mitochondrial leakage of cytochrome $c$. However, only cell viability and partially the fraction of apoptotic nuclei varied concomitantly with changes in AMPK activity, suggesting that AMPK is critical for metformin-mediated effects and regulates programmed cell death in a caspase-independent manner. Experiments with the inhibitors of JNK and p38 supports the role of these kinases in the drug-related inhibition of mitochondrial and extrinsic pathway of apoptosis.
\end{abstract}

Key words: metformin, apoptosis, astrocytes, oxygen-glucose deprivation.

\section{Introduction}

Metformin is an antihyperglycemic agent approved for treatment of type 2 diabetes. Its main mechanism of action focuses on the activation of AMP-activated protein kinase [36]. However, other molecular targets involving p38 mitogen-activated protein kinase (MAPK) and c-Jun N-terminal kinase (JNK) were identified as responsible for the effects of the drug in different experimental contexts [8,9,34]. As stroke is a major sequel of diabetes, epidemiological studies conducted in populations of diabetic patients analyzed the influence of metformin on the incidence of stroke and found that metformin reduced the risk of stroke $[7,30]$ and lowered 30-day mortality rates in patients with ischemic stroke [15]. Experimental studies further scrutinized metformin as a disease-modifying treatment in brain ischemia, and found that the drug improved both brain histological injury and functional outcome, as well as dissociated its protective effects from its anti-diabetic action $[1,16,20,31]$. Astrocytes fairly outnumber neurons, maintain the brain micro-environment, regulate synaptic activity and support the integrity of the blood-brain barrier. It is hypothesized that suppression of resident astrocytes' death may limit brain architectural damage, 
subsequent reactive astrogliosis and formation of glial scar, as well as improve neurological outcome following stroke [2]. Since astrocytes are relatively resistant to simulated ischemia, majority of cells undergo programmed cell death. Importantly, astrocytes express both organic cation transporter 3 (OCT3) and AMPK, the intracellular transporter for metformin $[6,35]$ and its main molecular target $[11,36]$, respectively. Furthermore, metformin was found to activate AMPK and alter metabolism in mouse cerebral cortex astrocytes [33]. Previously, we found that AMPK is activated in astrocytes during combined oxygen and glucose deprivation (OGD), and that both pharmacological and genetic inhibition of AMPK lead to profound decrease in cell viability [11]. Therefore, astrocytes may be the cellular effector of metformin in stroke. In this study, we examined the protective potential of metformin against ischemia-simulating conditions in primary rat cortical astrocytes. Our analysis involved cell viability, ratio of cells undergoing programmed cell death, and markers of both mitochondrial and common pathways of apoptosis. Finally, we investigated the role of AMPK, p38 and JNK p46/p54 kinases in the drug-mediated effects.

\section{Material and methods Reagents}

3-(4,5-dimethylthiazol-2-yl)-2,5-diphenyltetrazolium bromide (MTT), compound C (CC), SP600125 (SP), SB203580 (SB), Hoechst 33342 were purchased from Sigma-Aldrich (St. Louis, MO, USA). Dulbecco's modified Eagle's medium (DMEM), fetal bovine serum (FBS) and penicillin-streptomycin solution were purchased from Gibco (Carlsbad, CA, USA). Antibodies against phospho-AMPK $\alpha 1 / 2$ (Thr172), AMPK $\alpha 1 / 2$, acetyl-CoA carboxylase $\alpha$ (ACC $\alpha)$, phospho-ACC $\alpha$ (Ser78/Ser80), cytochrome $c$ and $\beta$-actin were obtained from Santa Cruz Biotechnology (Dallas, TX, USA). Antibodies against cleaved caspase $3, \mathrm{Bcl}-\mathrm{X}_{\mathrm{L}}$, p-JNK (Thr183/Thr185), JNK, p-p38 (Tyr180/Thr182) and p38 were obtained from Cell Signaling Technology (Danvers, MA, USA). ECL Prime Western Blotting Detection Reagent was purchased from GE Healthcare (Little Chalfont, UK).

\section{Cell culture}

Primary rat fetal-derived astrocytes were obtained as described previously [10], following approval by the Local Ethics Commission for Animal Experimentation.
Briefly, cerebral hemispheres were dissected, freed of the meninges, minced, and mechanically disrupted in DMEM culture medium supplemented with $1 \%$ of antibiotic-antimycotic solution [17]. The suspension was filtered through sterile nylon screening cloths with pore sizes of $70 \mu \mathrm{m}$ (first sieving) and $10 \mu \mathrm{m}$ (second sieving). The concentration of cells in suspension was adjusted to $1 \times 10^{6}$ cells $/ \mathrm{ml}$. For the MTT assay, the cells were seeded at $1 \times 10^{4}$ on 96 -well plates. For Hoechst 33342 staining, astrocytes were grown on coverslips covered with poly-D-lysine $(100 \mu \mathrm{g} / \mathrm{ml})$ at the density of $3 \times 10^{5} /$ dish. The cells destined for Western blot analysis were sieved onto plastic dishes of $100 \mathrm{~mm}$ in diameter at the density of $1 \times 10^{6} /$ dish. The culture medium initially contained $20 \% \mathrm{FBS}$, and after 4 days, was replaced with medium containing $10 \%$ FBS. After 14 days, the confluent cultures of astrocytes were deprived of microglia and oligodendrocytes by shaking at $150 \mathrm{rpm}$ with an orbital shaker for $3 \mathrm{~h}$. All experiments were performed on 21-day-old cultures and more than $98 \%$ of the cells were labeled with antibodies against glial fibrillary acidic protein [11].

\section{Oxygen-glucose deprivation and cell treatment}

Astrocytes, washed twice with serum and glucose-free DMEM, were subjected to ischemia-simulating conditions (OGD): $92 \% \mathrm{~N}_{2}, 5 \% \mathrm{CO}_{2}$ and $3 \% \mathrm{O}_{2}$ at $37^{\circ} \mathrm{C}$ (incubator Galaxy $48 \mathrm{R}$, Eppendorf Inc., USA) for 2-24 $\mathrm{h}$ [11]. To activate AMPK, the astrocytes were pre-incubated with metformin for $2 \mathrm{~h}$. To inhibit AMPK, the cells were pre-incubated with CC for $1 \mathrm{~h}$, and metformin was added for another 2 h. To inhibit JNK p46/p54 and p38 the cell cultures were pre-treated with SP and SB, respectively, for $1 \mathrm{~h}$. The studied compounds were present in the incubation medium during $12 \mathrm{~h}$ of OGD. Samples were processed immediately after OGD. Control cultures were placed in standard DMEM supplemented with $10 \%$ FBS and exposed to normoxia.

\section{Cell viability assay}

Cell viability was determined using MTT assay [23]. MTT $(0.25 \mathrm{mg} / \mathrm{ml})$ was added to the medium $3 \mathrm{~h}$ before the scheduled end of the experiment, and then the cultures were incubated at $37^{\circ} \mathrm{C}$ in proper conditions. After $3 \mathrm{~h}$, the formazan precipitate was dissolved in $100 \mu \mathrm{l}$ DMSO. Absorbance rates were 
measured at $570 \mathrm{~nm}$ using a microplate reader (Multiscan, Labsystems, Helsinki, Finland).

\section{Hoechst 33342 staining}

Apoptosis was determined by Hoechst 33342 staining. Astrocytes cultured on coverslips were fixed with $4 \%$ paraformaldehyde, and stained with $5 \mu \mathrm{g} / \mathrm{ml}$ Hoechst 33342. Cell nuclei analysis was conducted under fluorescence microscope and 20x objective (Nikon Corporation, Tokyo, Japan). The pictures were analyzed using ImageJ software $(1.48 \mathrm{v}$, $\mathrm{NIH}$, USA; http://imagej.nih.gov/ij/).

The number of apoptotic nuclei was determined on at least six randomly selected areas from three coverslips of every experimental group, each containing approximately 200 cells. The results were expressed as \% of apoptotic cells according to the equation: $\%$ of apoptotic cells $=($ apoptotic cells $) /($ all cells $) \times 100$.

\section{Western blotting}

The proteins were extracted with lysis buffer (50 mM Tris- $\mathrm{HCl}, \mathrm{pH} 7.4,150 \mathrm{mM} \mathrm{NaCl}, 0.5 \%$ Igepal, $0.1 \%$ sodium dodecyl sulfate [SDS], $10 \mu \mathrm{g} / \mathrm{ml}$ phenylmethylsulfonyl fluoride, $10 \mu \mathrm{g} / \mathrm{ml}$ leupeptin, $10 \mu \mathrm{g} / \mathrm{ml}$ pepstatin, and $10 \mu \mathrm{g} / \mathrm{ml}$ of heat-activated sodium orthovanadate). Cytosolic fractions were prepared as described previously [10]. The protein samples were loaded into 10\% SDS-PAGE, then the PVDF membranes were incubated overnight with

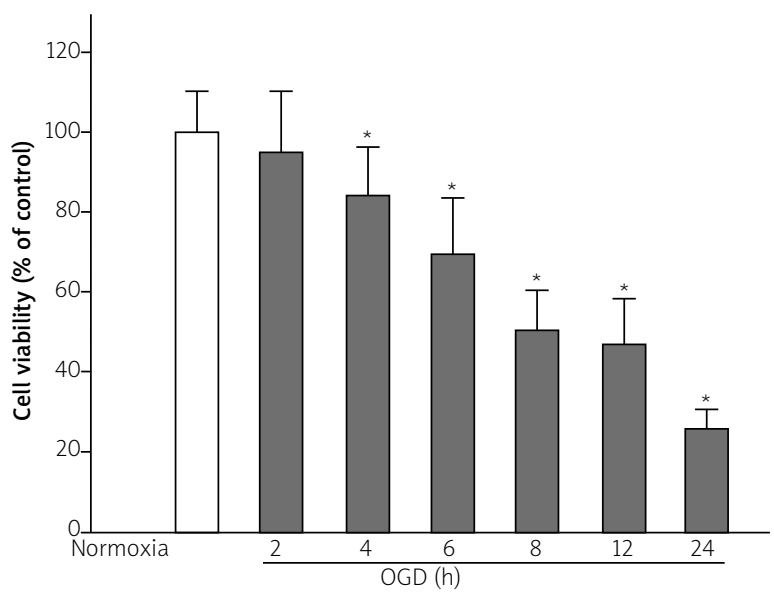

Fig. 1. Cell viability of astrocytes exposed to normoxia or 2-24 h of oxygen and glucose deprivation measured by MTT assay. Results are mean $\pm \mathrm{SD}(n=12) .{ }^{*} p<0.05$ vs. normoxia. the following polyclonal antibodies: phospho-AMPKa1/2 (1:150), AMPK $\alpha 1 / 2$ (1:150), ACC $\alpha(1: 200)$, phospho-ACC $\alpha(1: 200)$, cleaved caspase $3(1: 500)$, Bcl-X $(1: 250)$, cytochrome c $(1: 250), p$-JNK $(1: 200)$, JNK (1:200), p-p38 (Tyr180/Thr182, $1: 250)$, p38 $(1: 250), \beta$-actin $(1: 1000)$. Next, the membranes were incubated with appropriate HRP-conjugated secondary antibody $(1: 1000)$. The blots were developed using the ECL and detected with a ChemiDoc-IT 410 system (Ultra-Violet Products Ltd, Upland, CA, USA). Semi-quantitative analysis was performed with ImageJ (1.48v, NIH, USA; http://imagej.nih.gov/ij/).

\section{Statistical analysis}

All data are expressed as a mean \pm SD. Statistical analysis was performed using one-way analysis of variance (ANOVA) followed by the Bonferroni's post-hoc test. Differences were considered significant at $p<0.05$.

\section{Results}

\section{Oxygen-glucose deprivation induced cell viability loss}

We analyzed the time-dependent reduction of cell viability of astrocytes subjected to OGD and looked for

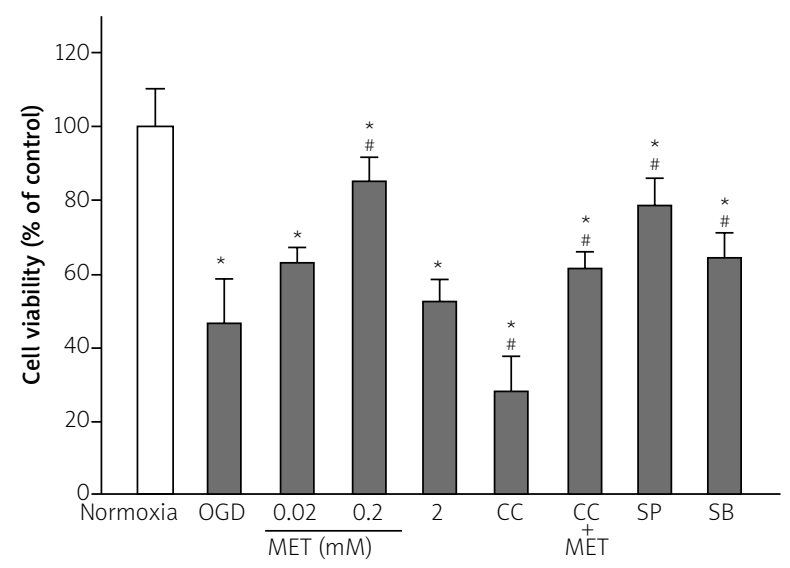

Fig. 2. Metformin (MET), SP600125 (SP) and SB203580 (SB) increase and compound C (CC) decreases the viability of astrocytes exposed to oxygen and glucose deprivation (OGD). Cells were exposed to normoxia or $12 \mathrm{~h}$ of OGD and treated with MET $(0.02-2 \mathrm{mM}), \mathrm{CC}(10 \mu \mathrm{M})$ with or without MET $(0.2 \mathrm{mM})$, SP $(10 \mu \mathrm{M})$ and SB $(10 \mu \mathrm{M})$ as described in the Material and methods section. Results are mean \pm SD $(n=12) .{ }^{*} p<0.05$ vs. normoxia; ${ }^{\#} p<0.05$ vs. OGD. 
$50 \%$ reduction in cell viability (Fig. 1). Gradual reduction of cell viability started after 4 hours of OGD and achieved $50 \%$ reduction after twelve hours.

\section{Metformin, SP and SB increase and CC decrease cell viability}

We analyzed the effect of logarithmically different concentrations of metformin on cell viability in the previously selected twelve-hour interval (Fig. 2). Metformin potently attenuated reductions in cell viability only at the concentration of $0.2 \mathrm{mM}$, and this concentration was chosen for subsequent analysis. The magnitude of the effect was comparable to that caused by the selective mitogen-activated protein kinases (MAPKs) inhibitors, SP (JNK inhibitor) and SB (p38 inhibitor). Importantly, the effect of metformin was nullified by CC - a cell-permeable, potent, reversible, and ATP-competitive pharmacological inhibitor of AMPK [36]. CC alone reduced cell viability, but metformin counteracted this effect. Based on published data, we decided to pre-incubate the cells for $1 \mathrm{~h}$ with CC, SP and SB (all at $10 \mu \mathrm{M})$, known to effectively inhibit AMPK, JNK and p38, respectively, in astrocytes [11,32].

\section{AMPK activation by metformin inhibits caspase- 3 cleavage}

The activity of AMPK is regulated by phosphorylation of the subunit at Thr172 by an upstream kinase

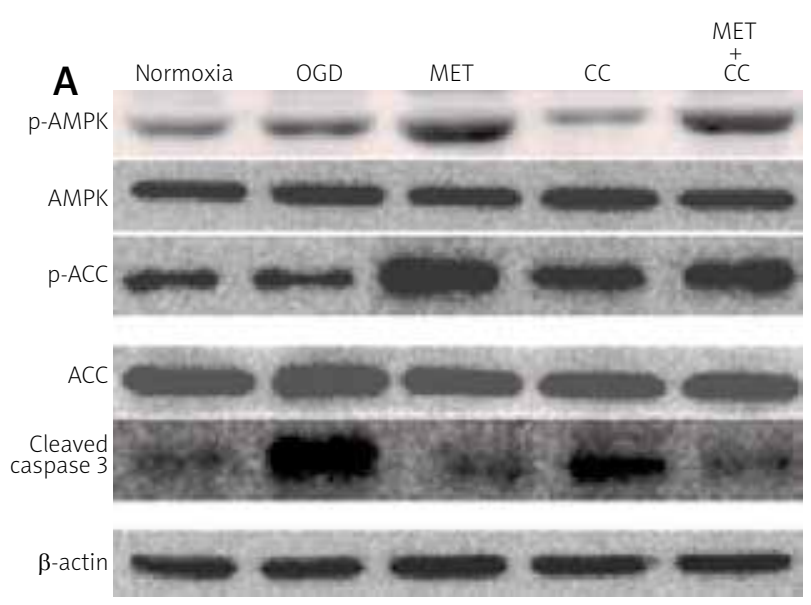

LKB1 [28]. A major downstream target of AMPK activation is ACC, a crucial enzyme in the regulation of fatty acid metabolism. Upon activation, AMPK enhances the phosphorylation and inactivation of ACC, limiting the anabolic process of fatty acid synthesis in times of energy deficiency [22]. Markers of AMPK activation ( $\mathrm{p}-\mathrm{AMPK}, \mathrm{p}-\mathrm{ACC}$ ) were unchanged 12 hours after OGD (Fig. 3). Metformin increased both phosphorylation of AMPK and its substrate ACC, but pharmacological inhibitor of AMPK attenuated phosphorylation of ACC in comparison with metformin. CC alone clearly decreased phosphorylation of AMPK, and this effect was counteracted by metformin. Therefore, the concentration of the compounds used were adequate to study AMPK-dependence of variables. Furthermore, it should be noted that total AMPK and ACC levels were unchanged in all experimental groups. We analyzed the activity of the executive caspase 3 with the expression of cleaved caspase 3 (Fig. 3). Predictably, 12 hours of OGD increased the activity of caspase 3 around threefold. Metformin attenuated the increase in the expression of cleaved caspase 3, and pharmacolog-

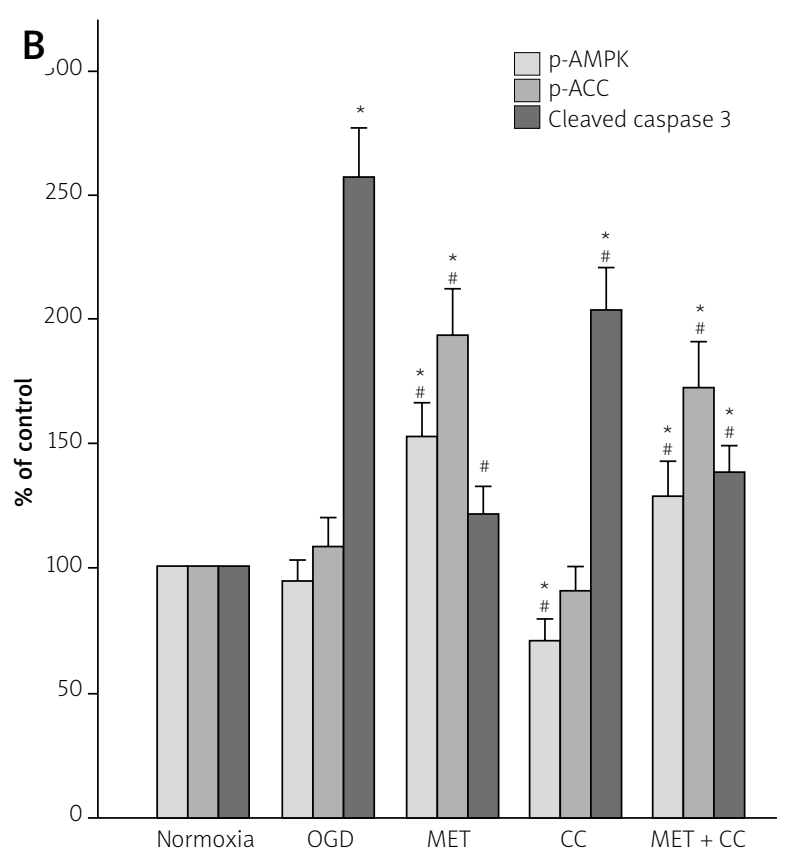

Fig. 3. Metformin activates AMP-activated protein kinase (AMPK) and inhibits caspase-3 cleavage. A) The representative images of western blot analysis of p-AMPK, AMPK, p-ACC, ACC and cleaved caspase 3 in astrocytes exposed to normoxia or $12 \mathrm{~h}$ of oxygen and glucose deprivation (OGD) and treated with $0.2 \mathrm{mM}$ metformin (MET) and $10 \mu \mathrm{M}$ compound C (CC) with or without MET, as described in the Material and methods section. $\beta$-actin was used as the loading control. B) Densitometric analyses of p-AMPK, p-ACC and cleaved caspase 3 normalized to $\beta$-actin. Results are mean \pm SD $(n=3) .{ }^{*} p<0.05$ vs. normoxia; ${ }^{*} p<0.05$ vs. OGD. 

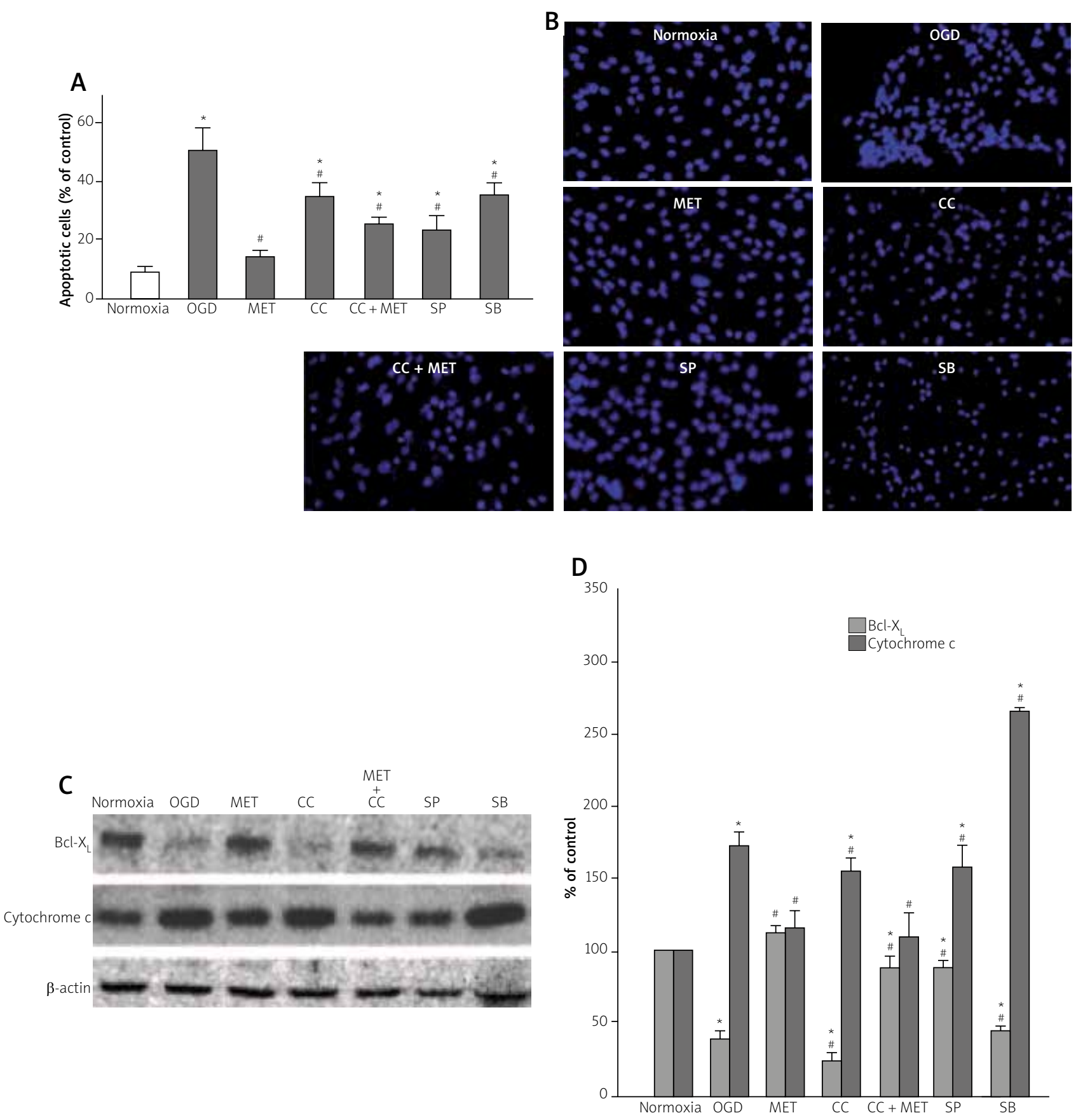

Fig. 4. Effect of metformin on oxygen and glucose deprivation (OGD)-induced apoptosis. Astrocytes were exposed to normoxia or $12 \mathrm{~h} \mathrm{OGD}$ and treated with $0.2 \mathrm{mM}$ metformin (MET), $10 \mu \mathrm{M}$ compound $\mathrm{C}$ (CC) with or without MET, $10 \mu \mathrm{M} \mathrm{SP}$ and $10 \mu \mathrm{M}$ SB, as described in the Material and methods section. The level of apoptosis was determined by Hoechst staining (A). Data are shown as a percentage relation of the apoptotic nuclei to the total amount of nuclei in the field and are the mean \pm SD of six randomly selected areas from three culture dishes in three separate experiments; ${ }^{*} p<0.05$ vs. normoxia; ${ }^{*} p<0.05$ vs. OGD. Representative images are presented in (B). C) The representative images of western blot analysis of $B c l-X_{L}$ in whole cell lysates and cytochrome $c$ expression in cytosolic fractions. $\beta$-actin was used as the loading control. D) Densitometric analyses of $\mathrm{BCl}-\mathrm{X}_{\mathrm{L}}$ and cytochrome $\mathrm{c}$ normalized to $\beta$-actin. Results are mean $\pm \mathrm{SD}$ $(n=3) .{ }^{*} p<0.05$ vs. normoxia; ${ }^{*} p<0.05$ vs. OGD. 
ical inhibitor of AMPK reversed this effect. CC alone increased cleaved caspase 3 expression.

\section{Effect of metformin, CC, SP, and SB on apoptosis}

Programmed cell death involves chromatin condensation with the appearance of pyknotic nuclei that morphologically distinguish apoptotic from necrotic cells. We analyzed the effect of metformin on the fraction of pyknotic nuclei with Hoechst 33342 stain (Fig. 4A-B). As expected, $12 \mathrm{~h}$ OGD increased fraction of apoptotic nuclei to around 50\%. Metformin normalized fraction of apoptotic nuclei, and the effect was partially reversed with CC. Importantly, CC alone had no positive effect on the fraction of apoptotic nuclei in comparison with normoxia. Predictably, inhibitors of p38 MAPK and JNK reduced the apoptosis, but the effect was weaker than that observed with metformin. $\mathrm{Bcl}-\mathrm{X}_{\perp}$ is an anti-apoptotic protein, which inhibits pore formation in mitochondrial membrane, and prevents the release of cytochrome $c$ in mitochondrial pathway of apoptosis. Predictably, the expression of Bcl- $X_{L}$ and cytosolic cytochrome $c$ varied inversely proportional in our model. The former was reduced and the latter increased after 12 hours of OGD. Metformin attenuated changes in the expression of these markers caused by OGD, but the pharmacological inhibitor of AMPK did not change this effect. CC alone slightly decreased the expression of both Bcl- $X_{L}$ and cytosolic cytochrome $c$. The effect of SP and SB on expression of these proteins was weaker than that of metformin (Fig. 4C-D).

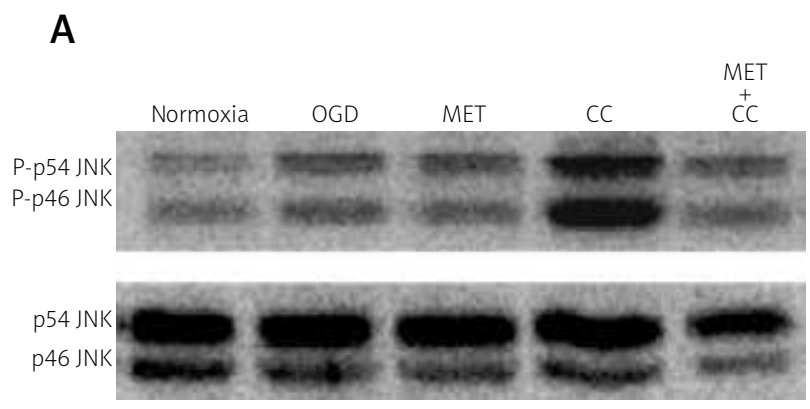

\section{Effect of metformin and CC on JNK and p38 MAPK signaling pathways}

The phosphorylation of JNK and p38 MAPK was enhanced by OGD treatment (Figs. 5 and 6). Metformin decreased OGD-induced activation of p54 JNK and p38 MAPK while the phosphorylation of p46 JNK remained unaffected. The treatment of ischemic astrocytes with CC did not attenuate the effect of metformin on these variables. Compound $C$ alone did not affect the phosphorylation level of p54 JNK, but decreased the phosphorylation of p38 MAPK (Figs. 5 and 6).

\section{Discussion}

AMPK is activated by cellular stressors such as reactive oxygen species (ROS) and depleted intracellular energy, and is involved in the regulation of cellular metabolism and death [14]. Previously, we have shown OGD activates AMPK in astrocytes, and AMPK activation prevents decline in cell viability [11].

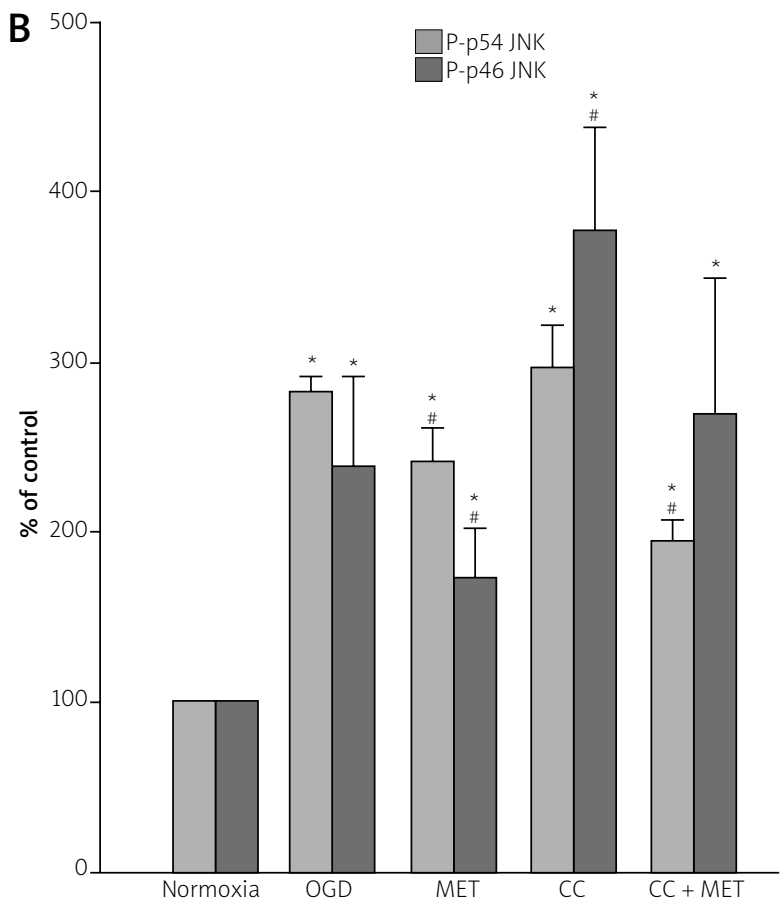

Fig. 5. Metformin attenuates oxygen and glucose deprivation (OGD)-induced activation of JNK. A) The representative images of western blot analysis of phosphorylated JNK (P-p54 JNK and P-p46 JNK) and total JNK in astrocytes exposed to normoxia or $12 \mathrm{~h} \mathrm{OGD}$ and treated with $0.2 \mathrm{mM}$ metformin (MET) and $10 \mu \mathrm{M} \mathrm{com-}$ pound C (CC) with or without MET, as described in the Material and methods section. B) Densitometric analyses of P-p54 JNK and P-p46 JNK normalized to p54 JNK and p46 JNK, respectively. Results are mean \pm SD $(n=3)$. ${ }^{*} p<0.05$ vs. normoxia; ${ }^{\#} p<0.05$ vs. OGD. 
A

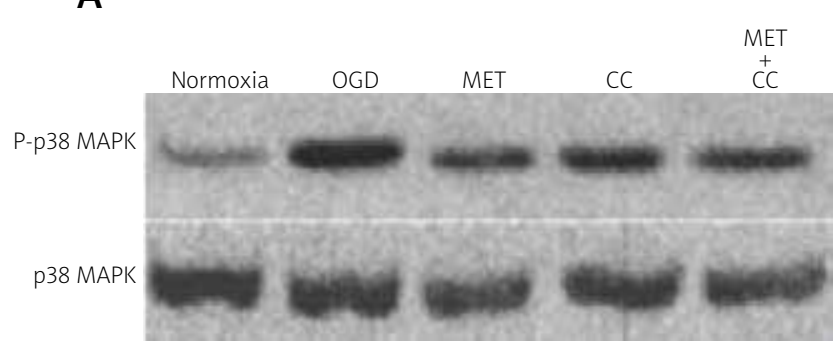

B

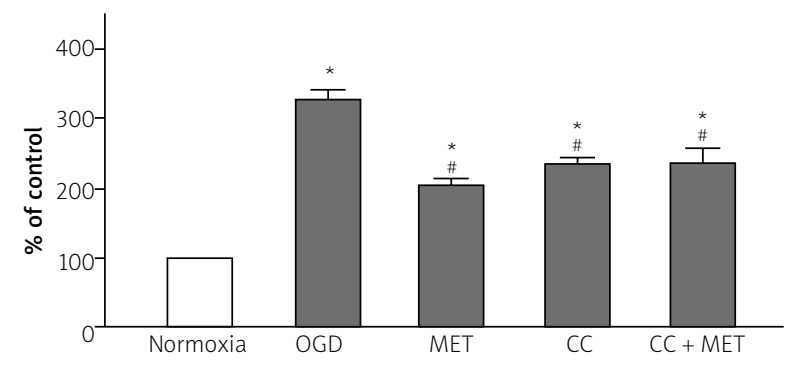

Fig. 6. Metformin attenuates oxygen and glucose deprivation (OGD)-induced activation of p-38 MAPK. A) The representative images of western blot analysis of phosphorylated p38 MAPK and total p38 MAPK in astrocytes exposed to normoxia or $12 \mathrm{~h} \mathrm{OGD}$ and treated with $0.2 \mathrm{mM}$ metformin (MET) and $10 \mu \mathrm{M}$ compound $\mathrm{C}$ (CC) with or without MET, as described in the Material and methods section. B) Densitometric analyses of P-p38 MAPK normalized to p38 MAPK. Results are mean \pm SD $(n=3) .{ }^{*} p<0.05$ vs. normoxia; $\# p<0.05$ vs. OGD.

However, the activation of AMPK lasts between 4 and 6 hours despite persistence of the stimulus, implying desensitization of AMPK activating pathway to OGD, and thereafter cell viability declines. Here, metformin maintained activation of AMPK up to 12 hours of OGD (Fig. 3), and similarly AMPK prevented decline in cell viability, as evidenced by the results obtained with compound $C$. Although inhibitors of JNK and p38 prevented decline in cell viability (Fig. 2) and metformin potently inhibited JNK (Fig. 5) and p38 (Fig. 6), inhibition of AMPK attenuated the effect of metformin (Figs. 2 and 3) pointing that at least temporal AMPK activation is crucial for astrocyte survival under OGD. AMPK oxidizes and loses its function under cellular stress, and may regain its capabilities in a redox-sensitive way [27]. Since JNK and p38 MAPK are redox-sensitive kinases [19], and metformin inhibited their activation in AMPK-independent manner (Figs. 3, 5 and 6), metformin might reduce formation of ROS, and thereby prevent deactivation of AMPK. Chai et al. [5] showed that metformin activates thioredoxin 1, and Shao et al. [27] showed that thioredoxin 1 prevents AMPK oxidation. Moreover, thioredoxin 1 was shown to inhibit ASK-1, which is upstream activator of JNK and p38 [21]. Therefore, it is plausible that metformin maintained AMPK activity by up-regulating thioredoxin 1 in our experimental model. Prolonged activation of JNK and p38 kinases inhibits Bcl-2 and its homologues, such as $\mathrm{Bcl}-\mathrm{X}_{\mathrm{L}}$, which results in cytochrome $\mathrm{c}$ release and the activation of the intrinsic pathway of apoptosis [29]. Accordingly, metformin inhibited JNK and p38 kinases, maintained the expression of $\mathrm{BCl}-\mathrm{X}_{\mathrm{L}}$ under OGD, prevented cytochrome c release, subsequent activation of the executive caspase 3 , and reduced the fraction of cells undergoing programmed cell death (Figs. 3-6). Importantly, the activity of p38 and JNK did not depend on AMPK activation, as shown by no effect of AMPK inhibition on these variables (Figs. 3-5). Pharmacological inhibitors of p38 and JNK affected the expression of $\mathrm{BCl}-\mathrm{X}_{\mathrm{L}}$, cytochrome $c$ release, and the fraction of cells undergoing programmed cell death (Figs. 4 and 5), further supporting the role of JNK and p38 kinases in the effects of metformin. The inhibition of the apoptosis, that is caspase-dependent programmed cell death, could rely on AMPK, as shown by increased cleaved caspase 3 expression (Fig. 3) and partially increased fraction of cells undergoing programmed cell death in compound C-treated samples (Fig. 4). Although caspase 3 activity did not vary with changes in AMPK activity, the fraction of cells undergoing programmed cell death varied with changes in the latter variable (Figs. 3 and 4). The action of AMPK may involve direct effect on AIF, BNIP-3, endonuclease G [4] and autophagy [18]. Autophagy is especially viable candidate to explain AMPK-mediated effects, because the process involves removal of damaged organelles and subsequent nutrient generation [11]. Therefore, it may lessen the strain caused by hypoxic injury and nutrient deprivation during OGD, and does not involve direct action on one of the redundant cell death pathways. Pharmacokinetics of metformin may limit translation of our results into either animal models or clinical scenarios, because the drug was protective only at the $0.2 \mathrm{mM}$, which is above 
the concentration found in the brain [24]. However, the concentration range of $\mathrm{mM}$ is used in most studies on metformin. Because metformin alters cellular signaling acting on mitochondria [25], no effect of the drug at the concentration range of $20 \mu \mathrm{M}$ may result from the insufficient time for the compound to reach its primary site of action. Since astrocytes express OCTs [26,35], and metformin is one of the substrates for OCTs [12], it is possible that the drug is pumped out of the cells, and cannot reach its cellular target. Conversely, during blood-brain barrier disruption, astrocytes expressing OCTs may concentrate metformin [6]. The loss of the effect at the concentration range of $\mathrm{mM}$ may be interpreted as a sign of drug toxicity, especially as metformin inhibits the complex I of the mitochondrial respiratory chain. Similar to our results showing bimodal effects of metformin, Jiang et al. [16] showed protective effects of acute metformin administration at a dose of $10 \mathrm{mg} / \mathrm{kg}$ in the permanent middle cerebral artery occlusion (MCAO) model, but Harada et al. [13] demonstrated toxic effects of acute metformin intracerebroventricular administration in transient MCAO at a dose of 25 and $100 \mu \mathrm{g} /$ mouse. In conclusion, metformin improved cell viability chiefly acting on AMPK, reduced the fraction of cells undergoing programmed cell death partially depending on AMPK activity and inhibited apoptosis independently of AMPK. The latter effect is consistent with the attenuation of JNK p54 and p38 MAPK pathways, the increase in the expression of apoptosis inhibitor, $\mathrm{BCl}-\mathrm{X}_{\mathrm{L}}$, and the attenuation of endogenous, that is mitochondrial, pathway of apoptosis. Our results regarding cell viability and programmed cell death suggest cross-talk between AMPK, JNK and p38 MAPK, the existence of redundant cell death pathways in astrocytes, and the involvement of autophagy that lessens the burden of OGD. Further research is required to clarify these issues in the protective effect of metformin.

\section{Acknowledgments}

The work was supported by grant No. N N401 072139 (B.G.) from the Ministry of Sciences and Higher Education, Warsaw, Poland.

\section{Disclosure}

The authors report no conflict of interest.

\section{References}

1. Ashabi G, Khalaj L, Khodagholi F, Goudarzvand M, Sarkaki A. Pre-treatment with metformin activates Nrf2 antioxidant pathways and inhibits inflammatory responses through induction of AMPK after transient global cerebral ischemia. Metab Brain Dis 2014; 30: 747-754.

2. Barreto G, White RE, Ouyang Y, Xu L, Giffard RG. Astrocytes: targets for neuroprotection in stroke. Cent Nerv Syst Agents Med Chem 2011; 11: 164-173.

3. Bradford MM. A rapid and sensitive method for the quantification of microgram quantities of protein utilizing the principle of protein dye binding. Anal Biochem 1976; 72: 248-251.

4. Broughton BR, Reutens DC, Sobey CG. Apoptotic mechanisms after cerebral ischemia. Stroke 2009; 40: e331-9.

5. Chai TF, Hong SY, He H, Zheng L, Hagen T, Luo Y, Yu FX. A potential mechanism of metformin-mediated regulation of glucose homeostasis: inhibition of Thioredoxin-interacting protein (Txnip) gene expression. Cell Signal 2012; 24: 1700-1705.

6. Chen EC, Liang X, Yee SW, Geier EG, Stocker SL, Chen L, Giacomini KM. Targeted disruption of organic cation transporter 3 attenuates the pharmacologic response to metformin. Mol Pharmacol 2015; 88: 75-83.

7. Cheng YY, Leu HB, Chen TJ, Chen CL, Kuo CH, Lee SD, Kao CL. Metformin-inclusive therapy reduces the risk of stroke in patients with diabetes: a 4-year follow-up study. J Stroke Cerebrovasc Dis 2014; 23: e99-105.

8. Conde de la Rosa L, Vrenken TE, Buist-Homan M, Faber KN. Moshage $\mathrm{H}$. Metformin protects primary rat hepatocytes against oxidative stress-induced apoptosis. Pharmacol Res Perspect 2015; 3: e00125.

9. Dai YL, Huang SL, Leng Y. AICAR and metformin exert AMPKdependent effects on INS-1E pancreatic $\beta$-cell apoptosis via differential downstream mechanisms. Int J Biol Sci 2015; 11: 1272-1280.

10. Gabryel B, Chalimoniuk M, Małecki A, Strosznajder JB. Effect of aniracetam on phosphatidylinositol transfer protein alpha in cytosolic and plasma membrane fractions of astrocytes subjected to simulated ischemia in vitro. Pharmacol Rep 2005; 57 : 664-669.

11. Gabryel B, Kost A, Kasprowska D, Liber S, Machnik G, Wiaderkiewicz R, Łabuzek K. AMP- activated protein kinase is involved in induction of protective autophagy in astrocytes exposed to oxygen-glucose deprivation. Cell Biol Int 2014; 38: 1086-1097.

12. Graham GG, Punt J, Arora M, Day RO, Doogue MP, Duong JK, Furlong TJ, Greenfield JR, Greenup LC, Kirkpatrick CM, Ray JE, Timmins P, Williams KM. Clinical pharmacokinetics of metformin. Clin Pharmacokinet 2011; 50: 81-98.

13. Harada S, Fujita-Hamabe W, Tokuyama S. The importance of regulation of blood glucose levels through activation of peripheral 5'-AMP-activated protein kinase on ischemic neuronal damage. Brain Res 2010; 1351: 254-263.

14. Hardie DG, Ross FA, Hawley SA. AMPK: a nutrient and energy sensor that maintains energy homeostasis. Nat Rev Mol Cell Biol 2012; 13: 251-262.

15. Horsdal HT, Mehnert F, Rungby J, Johnsen SP. Type of preadmission antidiabetic treatment and outcome among patients with 
ischemic stroke: a nationwide follow-up study. I Stroke Cerebrovasc Dis 2012; 21: 717-725.

16. Jiang T, Yu JT, Zhu XC, Wang HF, Tan MS, Cao L, Zhang QQ, Gao L, Shi JQ, Zhang YD, Tan L. Acute metformin preconditioning confers neuroprotection against focal cerebral ischaemia by pre-activation of AMPK-dependent autophagy. Br J Pharmacol 2014; 171: 3146-3157.

17. Juurlink BH, Hertz L: Plasticity of astrocytes in primary cultures: an experimental tool and a reason for methodological caution. Dev Neurosci 1985; 7: 263-277.

18. Kim J, Guan KL. Regulation of the autophagy initiating kinase ULK1 by nutrients: roles of mTORC1 and AMPK. Cell Cycle 2011; 10: 1337-1338.

19. Kim JS, Park ZY, Yoo YJ, Yu SS, Chun JS. p38 kinase mediates nitric oxide-induced apoptosis of chondrocytes through the inhibition of protein kinase $\mathrm{C}$ zeta by blocking autophosphorylation. Cell Death Differ 2015; 12: 201-212.

20. Li J, Benashski SE, Venna VR, McCullough LD. Effects of metformin in experimental stroke. Stroke 2010; 41: 2645-2652.

21. Liu H, Nishitoh H, Ichijo H, Kyriakis JM. Activation of apoptosis signal-regulating kinase 1 (ASK1) by tumor necrosis factor receptor-associated factor 2 requires prior dissociation of the ASK1 inhibitor thioredoxin. Mol Cell Biol 2000; 20: 2198-2208.

22. McCullough LD, Zeng Z, Li H, Landree LE, McFadden J, Ronnett GV. Pharmacological inhibition of AMP activated protein kinase provides neuroprotection in stroke. J Biol Chem 2005; 280: 20493-20502.

23. Mosmann T. Rapid colorimetric assay for cellular growth and survival: application to proliferation and cytotoxicity assays. J Immunol Methods 1983; 65: 55-63.

24. Łabuzek K, Suchy D, Gabryel B, Bielecka A, Liber S, Okopień B. Quantification of metformin by the HPLC method in brain regions, cerebrospinal fluid and plasma of rats treated with lipopolysaccharide. Pharmacol Rep 2010; 62: 956-965.

25. Owen MR, Doran E, Halestrap AP. Evidence that metformin exerts its anti-diabetic effects through inhibition of complex 1 of the mitochondrial respiratory chain. Biochem J 2000; 348: 607-614.

26. Perdan-Pirkmajer K, Pirkmajer S, Černe K, Kržan M. Molecular and kinetic characterization of histamine transport into adult rat cultured astrocytes. Neurochem Int 2012; 61: 415-422.

27. Shao D, Oka S, Liu T, Zhai P, Ago T, Sciarretta S, Li H, Sadoshima J. A redox-dependent mechanism for regulation of AMPK activation by Thioredoxin1 during energy starvation. Cell Metab 2014; 19: 232-245.

28. Son YO, Wang X, Hitron JA, Zhang Z, Cheng S, Budhraja A, Ding S, Lee JC, Shi X. Cadmium induces autophagy through ROSdependent activation of the LKB1-AMPK signaling in skin epidermal cells. Toxicol Appl Pharmacol 2011; 255: 287-296.

29. Tobiume K, Matsuzawa A, Takahashi T, Nishitoh H, Morita K, Takeda K, Minowa O, Miyazono K, Noda T, Ichijo H. ASK1 is required for sustained activations of JNK/p38 MAP kinases and apoptosis. EMBO Rep 2001; 2: 222-228.

30. UK Prospective Diabetes Study (UKPDS) Group. Effect of intensive blood-glucose control with metformin on complications in overweight patients with type 2 diabetes (UKPDS 34). Lancet 1998; 352: 854-865.
31. Venna VR, Li J, Hammond MD, Mancini NS, McCullough LD. Chronic metformin treatment improves post-stroke angiogenesis and recovery after experimental stroke. Eur J Neurosci 2014; 39: 2129-2138.

32. Wang J, Deng X, Zhang F, Chen D, Ding W. ZnO nanoparticleinduced oxidative stress triggers apoptosis by activating JNK signaling pathway in cultured primary astrocytes. Nanoscale Res Lett 2014; 9: 117.

33. Westhaus A, Blumrich EM, Dringen R. The Antidiabetic Drug Metformin Stimulates Glycolytic Lactate Production in Cultured Primary Rat Astrocytes. Neurochem Res 2017; 42: 294-305.

34. Yao XM, Ye SD, Xiao CC, Gu JF, Yang D, Wang S. Metformin alleviates high glucose-mediated oxidative stress in rat glomerular mesangial cells by modulation of p38 mitogen-activated protein kinase expression in vitro. Mol Med Rep 2015; 12: 520-526.

35. Yoshikawa T, Naganuma F, lida T, Nakamura T, Harada R, Mohsen AS, Kasajima A, Sasano H, Yanai K. Molecular mechanism of histamine clearance by primary human astrocytes. Glia 2013; 61: 905-916.

36. Zhou G, Myers R, Li Y, Chen Y, Shen X, Fenyk-Melody J, Wu M, Ventre J, Doebber T, Fujii N, Musi N, Hirshman MF, Goodyear LJ, Moller DE. Role of AMP-activated protein kinase in mechanism of metformin action. J Clin Invest 2011; 108: 1167-1174. 